\title{
Réponse à trois collègues ... et à quelques autres
}

\author{
Editorial de rentrée
}

Il y a manifestement de nombreux collègues qui attendent le répit de l'été pour prendre la plume et pour, ayant enfin un peu de temps, poser à la FMH ces questions qui les agacent depuis des mois - le courrier de juillet fait presque penser à l'avalanche de fin d'année!

Plusieurs de ces lettres interpellent le Comité central à propos de ses résultats politiques et expriment une impatience parfois tonique face à la lenteur des changements, face au rythme terriblement sage et tranquille avec lequel les choses évoluent.

Trois de ces lettres sont publiées aujourd'hui dans le «Forum du Comité central» (page 2031); elles sont suffisamment représentatives du courrier reçu cet été pour que je tente d’y répondre ici en reprenant les questions qu'elles soulèvent - elles intéresseront tout le monde, et elles permettront de préciser quelques points importants quant à notre méthode de travail.

- Concernant les statistiques des actes médicaux et de celles qui ont trait aux frais occasionnés par les patients de nos cabinets, notre situation se développe maintenant d'une façon tout à fait positive.

Nous disposons d'une base de données performante et de plus en plus riche ainsi que de gens expérimentés dans leur interprétation, au sein de l'entreprise NewIndex, et nous allons poursuivre ces efforts en consolidant ces outils au niveau de la FMH, dans les mois qui viennent; il s'agit pour le Comité central, dans le cadre de la nouvelle division Données, Démographie et Qualité du Secrétariat général, de disposer de façon efficace et reconnue des instruments d'analyse et de négociation dont l'ensemble du corps médical a besoin; c'est une priorité pour nous!

- La FMH, avec la collaboration de plusieurs collègues issus des sociétés cantonales, est également en train de concrétiser et de tester des outils d'analyse pour les fameux critères d'efficacité, d'adéquation et d'économicité dont un modèle de compensation des risques qui, enfin, prend en compte correctement la morbidité des patients, pour dépasser le misérable modèle actuel qui ne se fonde que sur le sexe et l'âge.

L'idée dans tout ce domaine de statistiques, de compensation des risques et de critères EAE est évidemment d'être prêts à réagir et à soutenir les collègues qui seraient l'objet d'attaques de la part des assureurs dans des procédures de remboursement injustifiées.

- Au sujet des limitations pour la facturation de certaines prestations, l'auteur de la lettre publiée aujourd'hui, le Dr J. Küttel, nous relance parce qu'en réponse à sa précédente interpellation, nous n'avions pas complètement pu lever le flou qui existe encore sur ces questions.

C'est vrai que sur ce point, les avis de la FMH et de santésuisse divergent. Nous n'allons donc pas pouvoir faire mieux que de réaffirmer notre propre position: le fait de tenir nos factures électroniques à disposition des assureurs, par l'intermédiaire d'un trustcenter par exemple, suffit à ce que les conditions pour la levée des limitations soient remplies - il n'est pas besoin que les assureurs de leur côté fassent concrètement appel à cette possibilité, respectivement qu'ils soient capables de le faire.

Nous pensons que vous devez vous prévaloir de ces explications pour exiger la levée des limitations dans vos factures. Attention, nous ne pouvons pas vous promettre que vous n'aurez pas à vous battre (en médecine comme ailleurs, il faut parfois accepter des risques!), mais nous pouvons par contre vous promettre que tant la FMH que votre société cantonale seront là pour vous soutenir et vous appuyer.

- Mais ce collègue demande aussi autre chose: que la situation soit clarifiée le $1^{\text {er }}$ janvier prochain, qu'on sache exactement où l'on en est, que les problèmes de transmission des données avec les assureurs soient réglés; là, il exige de nous quelque chose qu'on peut évidemment espérer, ... mais pas exiger! Nous sommes en négociations au sujet de la 
facturation électronique (et des limitations) avec plusieurs partenaires: santésuisse, mais aussi la CTM (Centrale des tarifs médicaux) pour les accidents, sans parler de l'OFSP qui essaie régulièrement de mettre son grain de sel un peu partout ...

Ces négociations sont donc en cours; vous imaginez bien que l'un des moyens de pression que nos partenaires utilisent volontiers est l'urgence, l'imminence des échéances à venir; nous ne sommes cependant pas prêts à accepter, sous prétexte d'urgence, des solutions bâtardes, et c'est simplement pour cela que nous n'avons pas encore, malgré le temps qui passe, de réponses définitives à ces questions.

C'est une décision explicite du Comité central de ne plus se laisser mettre sous pression, de négocier dorénavant sans hésiter à utiliser à fond les textes juridiques existants, et à faire preuve de fermeté: c'est de cette manière que nous pensons refléter le mieux les attentes de nos membres.

- La troisième lettre que nous publions pose la question des révisions tarifaires, et là aussi, c'est un domaine que nous sommes en train de faire évoluer, car l'inertie actuelle du système est simplement intolérable - quand bien même elle est ancrée dans les contrats que nous avons été amenés à signer à l'époque.

Ces contrats exigent l'unanimité entre la $\mathrm{FMH}, \mathrm{H+}$, santésuisse et la Centrale tarifaire LAA pour toute adaptation de la structure TARMED, ce qui est à la fois une sécurité pour nous ... et un facteur de blocage considérable. Et puis, cela mène bien sûr à des marchandages de bazar qui vont forcément menacer la cohérence du tarif; que faire de cette exigence d'unanimité est donc une discussion en cours entre les partenaires de TARMED Suisse.

- Par ailleurs, la prétention du Département de l'intérieur à contrôler chaque nouvelle version du TARMED avant de «l'agréer», outre qu'elle n'a aucun fondement légal, prend chaque fois presque six mois; nous avons pris l'option de ne plus nous soumettre à cette exigence injustifiée et de simplement informer le DFI et l'OFSP des modifications apportées d'une version à l'autre mais cette option n'est pas encore partagée par tous les membres de TARMED Suisse, notamment les assureurs-accident.

- Enfin, cette troisième lettre met en question le résultat des négociations, en l'occurrence sur le prix des prises de sang, ... et là, il faut bien dire que nous avons hésité, entre accepter un compromis et provoquer une rupture; sachant qu'en cas de rupture, c'est l'autorité administrative qui prend le relais et fixe les nombres de points, et vu les attaques de l'administration, justement, sur le laboratoire au cabinet, nous avons pris l'option du compromis.

Dans d'autres situations récentes, au contraire, nous avons été jusqu'à la rupture pour ne pas bafouer des principes que nous trouvons essentiels.

Il s'agit à chaque négociation d'une décision politique dans laquelle, vous pouvez en être certains, la situation concrète et les attentes des membres de la FMH ont une place centrale, fondamentale.

Bref, je crois que le Comité central est tout à fait conscient que les résultats que nous obtenons ne peuvent pas toujours être complètement à la hauteur des espoirs de nos membres. Vous devez cependant être bien certains que nous allons chaque fois le plus loin possible, en évaluant au mieux les intérêts du corps médical et en respectant nos principes - car nous n'avons finalement pas d'autres intérêts à défendre que ceux des médecins!

C'est cette relation de confiance entre nous, entre le Comité central et le corps médical, que je souhaite continuer à promouvoir dans l'année qui s'ouvre; cette relation de confiance est une force considérable pour nous toutes et tous.

Jacques de Haller, président de la FMH 


\title{
Antwort an drei Kollegen - und einige andere
}

\author{
Zum Wiederbeginn nach der Sommerpause
}

Offensichtlich warten viele Kolleginnen und Kollegen auf die Sommerpause, wo sie Zeit haben, zur Feder zu greifen und der FMH die Fragen zu stellen, die ihnen seit Monaten unter den Nägeln brennen: Die Zuschriften im Juli erinnern fast an die Postlawine von Ende Jahr!

In mehreren Briefen wird der Zentralvorstand auf seine politischen Ergebnisse angesprochen, und manchmal kommt angesichts des langsamen Wandels und der schrecklich braven und zähen Entwicklung grosse Ungeduld zum Ausdruck.

In dieser Ausgabe werden drei dieser Briefe im «Forum des Zentralvorstands» veröffentlicht (siehe Seite 2031). Sie sind unter den eingegangenen Zuschriften dieses Sommers so repräsentativ, dass ich versuchen möchte, an dieser Stelle auf die darin aufgeworfenen Fragen einzugehen. Diese Themen betreffen alle und erlauben es, einige wichtige Aspekte unserer Arbeitsmethode zu erläutern.

- Bei den Statistiken über die medizinischen Leistungen und die Kosten unserer Praxispatientinnen/-patienten entwickelt sich die Situation nun klar positiv.

Wir verfügen über eine leistungsfähige und immer reichhaltigere Datenbank sowie über Fachleute mit Erfahrung bei deren Interpretation, im Rahmen der Firma NewIndex. Diese Anstrengungen werden wir fortsetzen, und zwar durch eine Konsolidierung dieser Hilfsmittel auf der Ebene der FMH in den kommenden Monaten. Der Zentralvorstand soll im Rahmen der neuen Abteilung Daten, Demographie und Qualität im Generalsekretariat auf die von der gesamten Ärzteschaft benötigten, effizienten und anerkannten Analyse- und Verhandlungsinstrumente zurückgreifen können. Für uns ist diese Aufgabe prioritär.

- Die FMH konkretisiert und testet ausserdem in Zusammenarbeit mit mehreren Kolleginnen und Kollegen aus den kantonalen Ärztegesellschaften Analysehilfsmittel in bezug auf die berühmten WZW-Kriterien (Wirksamkeit, Zweckmässigkeit und Wirtschaftlichkeit). Dazu gehört ein Risikoausgleichsmodell, das endlich die Morbidität der Patientinnen und Patienten korrekt berück- sichtigt und das erbärmliche aktuelle Modell ersetzen könnte, das sich ausschliesslich auf Geschlecht und Alter stützt.

Die Idee hinter dieser Arbeit im Bereich Statistiken, Risikoausgleich und WZW-Kriterien ist es, dass wir bereit sind, zu reagieren und die Kolleginnen und Kollegen zu unterstützen, die von den Versicherern im Rahmen ungerechtfertigter Rückforderungsverfahren möglicherweise angegriffen werden.

- Was die Limitationen bei der Abrechnung bestimmter Leistungen anbelangt, so haben wir vom Autor eines heute veröffentlichten Briefes, Dr. J. Küttel, eine Nachfrage erhalten, weil wir mit unserer Antwort auf seine ursprüngliche Frage die Unklarheiten rund um dieses Thema nicht vollständig beseitigen konnten.

In dieser Angelegenheit vertreten die FMH und santésuisse in der Tat unterschiedliche Ansichten. Wir können also nicht mehr tun, als unseren Standpunkt zu bekräftigen: Wenn wir unsere elektronischen Rechnungen den Versicherern zur Verfügung stellen, z.B. über ein Trustcenter, sind die Bedingungen für die Aufhebung der Limitationen erfüllt. Es ist nicht nötig, dass die Versicherer ihrerseits diese Möglichkeit konkret nutzen bzw. dass sie dazu in der Lage sind.

Wir sind der Meinung, dass Sie dieses Argument geltend machen sollten, um die Aufhebung der Einschränkungen in Ihren Rechnungen zu fordern. Wir können Ihnen jedoch nicht versprechen, dass dies kampflos $\mathrm{zu}$ erreichen ist. (Wie anderswo muss man auch in der Medizin manchmal Risiken eingehen!) Wir können Ihnen aber versprechen, dass die FMH und Ihre kantonale Ärztegesellschaft Sie unterstützen werden.

- Der genannte Kollege fordert aber noch etwas anderes: Die Situation müsse bis am 1. Januar 2006 geklärt sein, damit wir genau wissen, wo wir stehen, und die Probleme bei der Datenübertragung mit den Versicherern müssten gelöst sein. Dies kann man selbstverständlich hoffen - aber nicht fordern. 
Über das Thema elektronische Abrechnung (inklusive Limitationen) verhandeln wir mit mehreren Partnern: santésuisse, Zentralstelle für Medizinaltarife UVG (ZMT) und BAG, das sich immer wieder und überall einzumischen versucht ...

- Die Verhandlungen laufen also, und Sie können sich sicher vorstellen, dass unsere Partner uns gerne mit der Dringlichkeit und dem drohenden Ablauf von Fristen unter Druck setzen. Wir sind jedoch nicht bereit, unter dem Vorwand des Zeitdrucks faule Kompromisse einzugehen, und deshalb können wir trotz der verstreichenden Zeit noch keine definitiven Antworten auf diese Fragen geben.

Der Zentralvorstand will sich ausdrücklich nicht mehr unter Druck setzen lassen, er will bei künftigen Verhandlungen von Beginn weg bestehende juristische Texte ins Feld führen und Entschlossenheit beweisen. Wir sind der Auffassung, dass wir so den Erwartungen unserer Mitglieder am besten gerecht werden.

- Im dritten veröffentlichten Brief geht es um die Tarifrevisionen. Auch auf diesem Gebiet sind wir dafür besorgt, dass sich die Dinge entwickeln, denn die Trägheit des heutigen Systems ist schlicht unannehmbar - auch wenn sie sich auf Verträge stützt, die wir seinerzeit unterzeichnet haben.

Diese Verträge verlangen für jede Änderung an der TARMED-Struktur Einstimmigkeit zwischen der FMH, H+, santésuisse und der ZMT UVG. Dies bedeutet einerseits Sicherheit für uns, ist aber andererseits ein erheblicher Hemmschuh. Ausserdem führt dieser Umstand natürlich auch zu Kuhhandeln, die zwangsläufig die Kohärenz des Tarifs gefährden werden. Aus diesem Grund diskutieren die Partner von TARMED Suisse zur Zeit darüber, was aus dieser Einstimmigkeitsbedingung werden soll.

- Der Anspruch des Departements des Innern, jede neue TARMED-Version zu kontrollieren und dann zu «genehmigen», stützt sich im übrigen auf keinerlei gesetzliche Grundlage, und ausserdem dauert dieses Verfahren jedesmal fast sechs Monate. Wir haben uns entschieden, dieser ungerechtfertigten Forderung nicht mehr nachzukommen und das EDI sowie das BAG über die Änderungen von einer Version zur nächsten einfach zu informieren. Dieses Vorgehen stösst jedoch noch nicht bei allen Mitgliedern von TARMED Suisse auf Zustimmung, insbesondere bei den Unfallversicherern nicht.

- In diesem dritten Brief wird schliesslich ein Verhandlungsergebnis in Frage gestellt, konkret betrifft es den Preis der Blutentnahmen. Bei diesem Punkt haben wir in der Tat gezögert: Sollen wir einen Kompromiss akzeptieren oder einen Abbruch der Verhandlungen provozieren? Da im zweiten Fall die Behörden die Anzahl Punkte festlegen würden und angesichts der von ihnen gestarteten Angriffe auf die Praxislabors haben wir uns für den Kompromiss entschieden.

In anderen Situationen der jüngsten Zeit gingen wir bis zum Verhandlungsabbruch, weil wir Prinzipien, die für uns wesentlich sind, nicht verraten wollten.

Bei allen Verhandlungen sind politische Entscheidungen zu treffen. Wir können Ihnen versichern, dass die konkrete Situation und die Erwartungen der FMH-Mitglieder dabei einen zentralen, grundlegenden Platz einnehmen.

Kurz: Ich denke, dass sich der Zentralvorstand sehr wohl bewusst ist, dass die erzielten Ergebnisse die Hoffnungen unserer Mitglieder nicht immer vollumfänglich erfüllen können. Sie können sich aber darauf verlassen, dass wir jedesmal so weit wie möglich gehen, dass wir dabei die Interessen der Ärzteschaft weitestgehend berücksichtigen und uns an unsere Grundsätze halten. Schliesslich haben wir ja keine anderen Interessen zu vertreten als diejenigen der Ärztinnen und Ärzte!

Es liegt mir viel daran, diese Vertrauensbeziehung zwischen dem Zentralvorstand und der Ärzteschaft weiter zu pflegen und auszubauen sie ist eine grosse Stärke für uns alle.

Jacques de Haller, Präsident der FMH 